\title{
SVM-Maj: a majorization approach to linear support vector machines with different hinge errors
}

\author{
P. J. F. Groenen · G. Nalbantov • J. C. Bioch
}

Received: 3 November 2007 / Revised: 23 February 2008 / Accepted: 25 February 2008

Published online: 27 March 2008

(C) The Author(s) 2008

\begin{abstract}
Support vector machines (SVM) are becoming increasingly popular for the prediction of a binary dependent variable. SVMs perform very well with respect to competing techniques. Often, the solution of an SVM is obtained by switching to the dual. In this paper, we stick to the primal support vector machine problem, study its effective aspects, and propose varieties of convex loss functions such as the standard for SVM with the absolute hinge error as well as the quadratic hinge and the Huber hinge errors. We present an iterative majorization algorithm that minimizes each of the adaptations. In addition, we show that many of the features of an SVM are also obtained by an optimal scaling approach to regression. We illustrate this with an example from the literature and do a comparison of different methods on several empirical data sets.
\end{abstract}

Keywords Support vector machines - Iterative majorization - Absolute hinge error · Quadratic hinge error · Huber hinge error · Optimal scaling

Mathematics Subject Classification (2000) $\quad 90 \mathrm{C} 30 \cdot 62 \mathrm{H} 30 \cdot 68 \mathrm{~T} 05$

P. J. F. Groenen $(\varangle)$ · J. C. Bioch

Econometric Institute, Erasmus University Rotterdam,

P. O. Box 1738, 3000 DR, Rotterdam, The Netherlands

e-mail: groenen@few.eur.nl

J. C. Bioch

e-mail: bioch@few.eur.nl

G. Nalbantov

ERIM and Econometric Institute, Erasmus University Rotterdam,

P. O. Box 1738, 3000 DR, Rotterdam, The Netherlands

e-mail: nalbantov@few.eur.nl 


\section{Introduction}

An increasingly more popular technique for the prediction of two groups from a set of predictor variables is the support vector machines (SVM, see, e.g., Vapnik 2000). Although alternative techniques such as linear and quadratic discriminant analysis, neural networks, and logistic regression can also be used to analyze this data analysis problem, the prediction quality of SVMs seems to compare favorably with respect to these competing models. Another advantage of SVMs is that they are formulated as a well-defined optimization problem that can be solved through a quadratic program. A second valuable property of the SVM is that the derived classification rule is relatively simple and can be readily applied to new, unseen samples. A potential disadvantage is that the interpretation in terms of the predictor variables in nonlinear SVM is not always possible. In addition, the usual dual formulation of an SVM may not be so easy to grasp.

In this paper, we restrict our focus to linear SVMs. We believe that this paper makes several contributions on three themes. First, we offer a nonstandard way of looking at linear SVMs that makes the interpretation easier. To do so, we stick to the primal problem and formulate the SVM in terms of a loss function that is regularized by a penalty term. From this formulation, it can be seen that SVMs use robustified errors. Apart from the standard SVM loss function that uses the absolute hinge error, we advocate two other hinge errors, the Huber and quadratic hinge errors, and show the relation with ridge regression. Note that recently, Rosset and Zhu (2007) also discusses the use of different errors in SVMs including these two hinge errors.

The second theme of this paper is to show the connection between optimal scaling regression and SVMs. The idea of optimally transforming a variable so that a criterion is being optimized has been around for more than 30 years (see, e.g., Young 1981; Gifi 1990). We show that optimal scaling regression using an ordinal transformation with the primary approach to ties comes close to the objective of SVMs. We discuss the similarities between both approaches and give a formulation of SVM in terms of optimal scaling.

A third theme is to develop and extend the majorization algorithm of Groenen et al. (2007) to minimize the loss for any of the hinge errors. We call this general algorithm SVM-Maj. The advantage of majorization is that each iteration is guaranteed to reduce the SVM loss function until convergence is reached. As the SVM loss functions with convex hinge errors such as the quadratic and Huber hinge errors are convex, the majorization algorithm stops at a minimum after a sufficient number of iterations. For the case of the Huber and quadratic hinge, the SVM-Maj algorithm turns out to yield computationally very efficient updates amounting to a single matrix multiplication per iteration. Through SVM-Maj, we contribute to the discussion on how to approach the linear SVM quadratic problem.

Finally, we provide numerical experiments on a suite of 14 empirical data sets to study the predictive performance of the different errors in SVMs and compare it to optimal scaling regression. We also compare the computational efficiency of the majorization approach for the SVM to several standard SVM solvers.

Note that this paper is a significantly extended version of Groenen et al. (2007). 

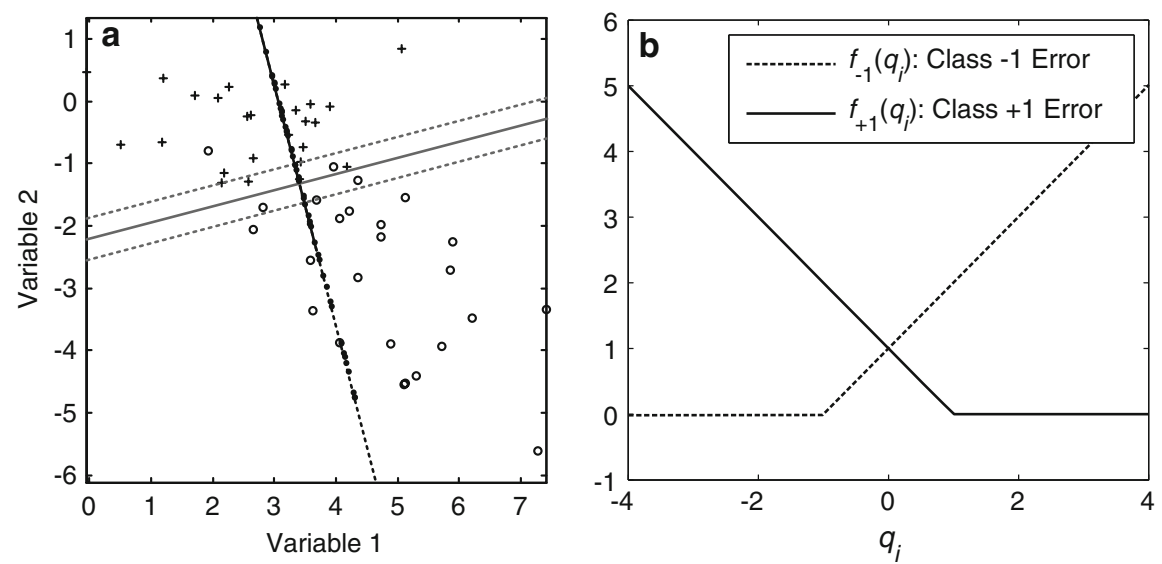

Fig. 1 a Projections of the observations in groups $1(+)$ and $-1(\mathrm{o})$ onto the line given by $w_{1}$ and $w_{2}$. b The absolute hinge error function $f_{1}\left(q_{i}\right)$ for class 1 objects (solid line) and $f_{-1}\left(q_{i}\right)$ for class -1 objects (dashed line)

\section{The SVM loss function}

Here, we present a rather non-mainstream view on explaining how SVM work. There is a quite close relationship between SVM and regression. We first introduce some notation. Let the matrix of quantitative predictor variables be represented by the $n \times m$ matrix $\mathbf{X}$ of $n$ objects and $m$ variables. The grouping of the objects into two classes is given by the $n \times 1$ vector $\mathbf{y}$, that is, $y_{i}=1$ if object $i$ belongs to class 1 and $y_{i}=-1$ if object $i$ belongs to class -1 . The exact labeling -1 and 1 to distinguish the classes is not important. The weights used to make a linear combination of the predictor variables is represented by the $m \times 1$ vector $\mathbf{w}$. Then, the predicted value $q_{i}$ for object $i$ is

$$
q_{i}=c+\mathbf{x}_{i}^{\prime} \mathbf{w}
$$

where $\mathbf{x}_{i}^{\prime}$ is row $i$ of $\mathbf{X}$ and $c$ is an intercept. As an illustrative example, consider Fig. 1a with a scatterplot of two predictor variables, where each row $i$ is represented by a point labelled ' + ' for the class 1 and 'o' for class -1 . Every combination of $w_{1}$ and $w_{2}$ defines a direction in this scatter plot. Then, each point $i$ can be projected onto this line. The main idea of the SVM is to choose this line in such a way that the projections of the points of class 1 are well separated from those of class -1 . The line of separation is orthogonal to the line with projections and the intercept $c$ determines where exactly it occurs. The length $\|\mathbf{w}\|$ of $\mathbf{w}$ has the following significance. If $\mathbf{w}$ has length 1 , that is, $\|\mathbf{w}\|=\left(\mathbf{w}^{\prime} \mathbf{w}\right)^{1 / 2}=1$, then Fig. 1a explains fully the linear combination (1). If w does not have length 1 , then the scale values along the projection line should be multiplied by $\|\mathbf{w}\|$. The dotted lines in Fig. 1a show all those points that project to the lines at $q_{i}=-1$ and $q_{i}=1$. These dotted lines are called the margin lines in SVMs. With three predictor variables, the objects are points in a three dimensional space, $\mathbf{w}$ still defines a direction, but all points that project on $\mathbf{w}$ at the same locations now 
form a plane and in higher dimensionality form a hyperplane. Thus, with more than two predictor variables, there will be a separation hyperplane and the margins are also hyperplanes. Summarizing, the SVM has three sets of parameters that determine its solution: (1) the weights normalized to have length 1 , that is, w/\|w $\|$, (2) the length of $\mathbf{w}$, that is, $\|\mathbf{w}\|$, and (3) the intercept $c$.

An error is counted in SVMs as follows. Every object $i$ from class 1 that projects such that $q_{i} \geq 1$ yields a zero error. However, if $q_{i}<1$, then the error is linear with $1-q_{i}$. Similarly, objects in class -1 with $q_{i} \leq-1$ do not contribute to the error, but those with $q_{i}>-1$ contribute linearly with $q_{i}+1$. Thus, objects that project on the wrong side of their margin contribute to the error, whereas objects that project on the correct side of their margin yield zero error. Figure $1 \mathrm{~b}$ shows the error functions for the two classes. Because of its hinge form, we call this error function the absolute hinge error.

As the length of $\mathbf{w}$ controls how close the margin lines are to each other, it can be beneficial for the number of errors to choose the largest $\|\mathbf{w}\|$ possible, so that fewer points contribute to the error. To control the $\|\mathbf{w}\|$, a penalty term that is dependent on $\|\mathbf{w}\|$ is added to the loss function. The penalty term also avoids overfitting of the data.

Let $G_{1}$ and $G_{-1}$ respectively denote the sets of class 1 and -1 objects. Then, the SVM loss function can be written as

$$
\begin{aligned}
& L_{\mathrm{SVM}}(c, \mathbf{w}) \\
& =\sum_{i \in G_{1}} \max \left(0,1-q_{i}\right)+\sum_{i \in G_{-1}} \max \left(0, q_{i}+1\right)+\lambda \mathbf{w}^{\prime} \mathbf{w} \\
& =\sum_{i \in G_{1}} f_{1}\left(q_{i}\right)+\sum_{i \in G_{-1}} f_{-1}\left(q_{i}\right)+\lambda \mathbf{w}^{\prime} \mathbf{w} \\
& =\text { Class } 1 \text { errors } \quad+\text { Class }-1 \text { errors }+ \text { Penalty for } \\
& \text { nonzero } \mathbf{w} \text {, }
\end{aligned}
$$

where $\lambda>0$ determines the strength of the penalty term. In this notation, the arguments $(c, \mathbf{w})$ indicate that $L_{\mathrm{SVM}}(c, \mathbf{w})$ needs to be minimized with respect to the arguments $c$ and w. For similar expressions, see Hastie et al. (2001) and Vapnik (2000). Note that (2) can also be expressed as

$$
L_{\mathrm{SVM}}(c, \mathbf{w})=\sum_{i=1}^{n} \max \left(0,1-y_{i} q_{i}\right)+\lambda \mathbf{w}^{\prime} \mathbf{w}
$$

which is closer to the expressions used in the SVM literature.

Once a solution $c$ and $\mathbf{w}$ is found that minimizes (2), we can determine how each object contributes to the error. Each object $i$ that projects on the correct side of its margin contributes with zero error to the loss. Therefore, these objects could be removed from the analysis without changing the minimum of (2) and the values of $c$ and $\mathbf{w}$ where this minimum is reached. The only objects determining the solution are those projecting on or at the wrong side of their margin thereby inducing error. Such objects are called support vectors as they form the fundament of the SVM solution. Unfortunately, these objects (the support vectors) are not known in advance and, therefore, the analysis needs to be carried out with all $n$ objects present in the analysis. It is the 
very essence of the SVM definition that error free data points have no influence on the solution.

From (2) it can be seen that any error is punished linearly, not quadratically. Therefore, SVMs are more robust against outliers than a least-squares loss function. The idea of introducing robustness by absolute errors is not new. For more information on robust multivariate analysis, we refer to Huber (1981), Vapnik (2000), and Rousseeuw and Leroy (2003). In the next section, we discuss two other error functions, one of which is robust.

In the SVM literature, the SVM loss function is usually presented as follows (Burges 1998):

$$
\begin{aligned}
L_{\text {SVMClas }}(c, \mathbf{w}, \xi)= & C \sum_{i \in G_{1}} \xi_{i}+C \sum_{i \in G_{2}} \xi_{i}+\frac{1}{2} \mathbf{w}^{\prime} \mathbf{w}, \\
\text { subject to } & 1+\left(c+\mathbf{w}^{\prime} \mathbf{x}_{i}\right) \leq \xi_{i} \text { for } i \in G_{-1} \\
& 1-\left(c+\mathbf{w}^{\prime} \mathbf{x}_{i}\right) \leq \xi_{i} \text { for } i \in G_{1} \\
& \xi_{i} \geq 0,
\end{aligned}
$$

where $C$ is a nonnegative parameter set by the user to weigh the importance of the errors represented by the so-called slack variables $\xi_{i}$. If object $i$ in $G_{1}$ projects at the correct side of its margin, that is, $q_{i}=c+\mathbf{w}^{\prime} \mathbf{x}_{i} \geq 1$, then $1-\left(c+\mathbf{w}^{\prime} \mathbf{x}_{i}\right) \leq 0$ so that the corresponding $\xi_{i}$ can be chosen as 0 . If $i$ projects on the wrong side of its margin, then $q_{i}=c+\mathbf{w}^{\prime} \mathbf{x}_{i}<1$ so that $1-\left(c+\mathbf{w}^{\prime} \mathbf{x}_{i}\right)>0$. Choosing $\xi_{i}=1-\left(c+\mathbf{w}^{\prime} \mathbf{x}_{i}\right)$ gives the smallest $\xi_{i}$ satisfying the restrictions in (4), (5), and (6). Therefore, $\xi_{i}=\max \left(0,1-q_{i}\right)$ and is a measure of error. For class -1 objects, a similar derivation can be made. Note that in the SVM literature (3) and (6) are often expressed more compactly as

$$
\begin{aligned}
L_{\mathrm{SVMClas}}(c, \mathbf{w}, \xi)= & C \sum_{i=1}^{n} \xi_{i}+\frac{1}{2} \mathbf{w}^{\prime} \mathbf{w}, \\
\text { subject to } \quad & y_{i}\left(c+\mathbf{w}^{\prime} \mathbf{x}_{i}\right) \leq 1-\xi_{i} \quad \text { for } \quad i=1, \ldots, n \\
& \xi_{i} \geq 0 .
\end{aligned}
$$

If we choose $C$ as $(2 \lambda)^{-1}$ then

$$
\begin{aligned}
& L_{\text {SVMClas }}(c, \mathbf{w}, \xi) \\
& =(2 \lambda)^{-1}\left(\sum_{i \in G_{1}} \xi_{i}+\sum_{i \in G_{-1}} \xi_{i}+2 \lambda \frac{1}{2} \mathbf{w}^{\prime} \mathbf{w}\right) \\
& =(2 \lambda)^{-1}\left(\sum_{i \in G_{1}} \max \left(0,1-q_{i}\right)+\sum_{i \in G_{-1}} \max \left(0, q_{i}+1\right)+\lambda \mathbf{w}^{\prime} \mathbf{w}\right) \\
& =(2 \lambda)^{-1} L_{\operatorname{SVM}}(c, \mathbf{w}) .
\end{aligned}
$$



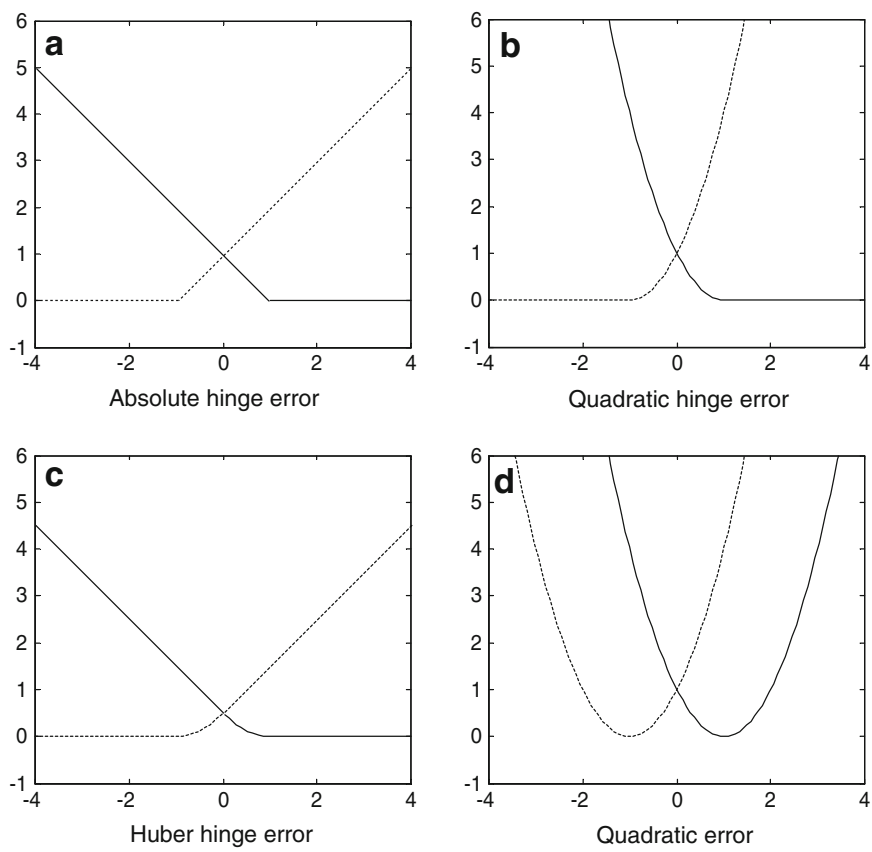

Fig. 2 Four error functions: a the absolute hinge error, $\mathbf{b}$ the quadratic hinge error, $\mathbf{c}$ the Huber hinge error, and $\mathbf{d}$ the quadratic error

showing that the two formulations (2) and (3) are exactly the same up to a scaling factor $(2 \lambda)^{-1}$ and yield the same $c$ and $\mathbf{w}$. The advantage of (2) lies in that it can be interpreted as a (robust) error function with a penalty. This quadratic penalty term is used for regularization much in the same way as in ridge regression, that is, to force the $w_{j}$ to be close to zero. The penalty is particularly useful to avoid overfitting. Furthermore, it can be easily seen that $L_{\mathrm{SVM}}(c, \mathbf{w})$ is a convex function in $c$ and $\mathbf{w}$ as all three terms are convex in $c$ and $\mathbf{w}$. The minimum of $L_{\mathrm{SVM}}(c, \mathbf{w})$ must be a global one as the function is convex and bounded below by zero. Note that the formulation in (3) allows the problem to be treated as a quadratic program. However, in Sect. 5, we optimize (2) directly by the method of iterative majorization.

\section{Other error functions}

An advantage of clearly separating error from penalty is that it is easy to apply other error functions. Instead of the absolute hinge error in Fig. 2a, we can use different definitions for the errors $f_{1}\left(q_{i}\right)$ and $f_{-1}\left(q_{i}\right)$. A straightforward alternative for the absolute hinge error is the quadratic hinge error, see Fig. 2b. This error simply squares the absolute hinge error, yielding the loss function

$$
L_{\mathrm{Q}-\operatorname{SVM}}(c, \mathbf{w})=\sum_{i \in G_{1}} \max \left(0,1-q_{i}\right)^{2}+\sum_{i \in G_{-1}} \max \left(0, q_{i}+1\right)^{2}+\lambda \mathbf{w}^{\prime} \mathbf{w},
$$


Table 1 Definition of error functions that can be used in the context of SVMs

\begin{tabular}{lll}
\hline Error & $f_{-1}\left(q_{i}\right)$ & \\
\hline Absolute hinge & $\max \left(0, q_{i}+1\right)$ & \\
Quadratic hinge & $\max \left(0, q_{i}+1\right)^{2}$ & if $q_{i} \leq k$ \\
Huber hinge & $h_{-1}\left(q_{i}\right)=(1 / 2)(k+1)^{-1} \max \left(0, q_{i}+1\right)^{2}$ & if $q_{i}>k$ \\
& $h_{-1}\left(q_{i}\right)=q_{i}+1-(k+1) / 2$ & \\
Quadratic & $\left(q_{i}+1\right)^{2}$ & \\
& & \\
Absolute hinge & $f_{+1}\left(q_{i}\right)$ & \\
Quadratic hinge & $\max \left(0,1-q_{i}\right)$ & if $q_{i} \leq-k$ \\
Huber hinge & $\max \left(0,1-q_{i}\right)^{2}$ & if $q_{i}>-k$ \\
& $h_{+1}\left(q_{i}\right)=1-q_{i}-(k+1) / 2$ & \\
Quadratic & $h_{+1}\left(q_{i}\right)=(1 / 2)(k+1)^{-1} \max \left(0,1-q_{i}\right)^{2}$ & \\
\hline & $\left(1-q_{i}\right)^{2}$ &
\end{tabular}

see also,Vapnik (2000) and Cristianini and Shawe-Taylor (2000). It uses the quadratic error for objects that have prediction error and zero error for correctly predicted objects. An advantage of this loss function is that both error and penalty terms are quadratic. In Sect. 5, we see that the majorizing algorithm is very efficient because in each iteration a linear system is solved very efficiently. A disadvantage of the quadratic hinge error is that outliers can have a large influence on the solution.

An alternative that is smooth and robust is the Huber hinge error, see Fig. 2c. This hinge error was called "Huberized squared hinge loss" by Rosset and Zhu (2007). Note that Chu et al. (2003) proposed a similar function for support vector regression. The definition of the Huber hinge is found in Table 1 and the corresponding SVM problem is defined by

$$
L_{\mathrm{H}-\mathrm{SVM}}(c, \mathbf{w})=\sum_{i \in G_{1}} h_{+1}\left(q_{i}\right)+\sum_{i \in G_{-1}} h_{-1}\left(q_{i}\right)+\lambda \mathbf{w}^{\prime} \mathbf{w} .
$$

The Huber hinge error is characterized by a linearly increasing error if the error is large, a smooth quadratic error for errors between 0 and the linear part, and zero for objects that are correctly predicted. The smoothness is governed by a value $k \geq-1$. The Huber hinge approaches the absolute hinge for $k \downarrow-1$, so that the Huber hinge SVM loss solution can approach the classical SVM solution. If $k$ is chosen too large, then the Huber hinge error essentially approaches the quadratic hinge function. Thus, the Huber hinge error can be seen as a compromise between the absolute and quadratic hinge errors. As we will see in Sect. 5, it is advantageous to choose $k$ sufficiently large, for example, $k=1$, as is done in Fig. 2c. A similar computational efficiency as for the quadratic hinge error is also available for the Huber hinge error.

In principle, any robust error can be used. To inherit as much of the nice properties of the standard SVM it is advantageous that the error function has two properties: (1) if the error function is convex in $q_{i}$ (and hence in $\mathbf{w}$ ), then the total loss function 
is also convex and hence has a global minimum that can be reached, (2) the error function should be asymmetric and have the form of a hinge so that objects that are predicted correctly induce zero error.

In Fig. $2 d$ the quadratic error is used, defined in Table 1. The quadratic error alone simply equals a multiple regression problem with a dependent variable $y_{i}=-1$ if $i \in G_{-1}$ and $y_{i}=1$ if $i \in G_{1}$, that is,

$$
\begin{aligned}
L_{\mathrm{MReg}}(c, \mathbf{w}) & =\sum_{i \in G_{1}}\left(1-q_{i}\right)^{2}+\sum_{i \in G_{-1}}\left(1+q_{i}\right)^{2}+\lambda \mathbf{w}^{\prime} \mathbf{w} \\
& =\sum_{i \in G_{1}}\left(y_{i}-q_{i}\right)^{2}+\sum_{i \in G_{-1}}\left(y_{i}-q_{i}\right)^{2}+\lambda \mathbf{w}^{\prime} \mathbf{w} \\
& =\sum_{i}\left(y_{i}-c-\mathbf{x}_{i}^{\prime} \mathbf{w}\right)^{2}+\lambda \mathbf{w}^{\prime} \mathbf{w} \\
& =\|\mathbf{y}-c \mathbf{1}-\mathbf{X} \mathbf{w}\|^{2}+\lambda \mathbf{w}^{\prime} \mathbf{w} .
\end{aligned}
$$

Note that for $i \in G_{-1}$ we have the equality $\left(1+q_{i}\right)^{2}=\left((-1)\left(1+q_{i}\right)\right)^{2}=\left(-1-q_{i}\right)^{2}=$ $\left(y_{i}-q_{i}\right)^{2}$. $L_{\text {MReg }}(c, \mathbf{w})$ has been extensively discussed in Suykens et al. (2002). To show that (9) is equivalent to ridge regression, we center the columns of $\mathbf{X}$ and use $\mathbf{J X}$ with $\mathbf{J}=\mathbf{I}-n^{-1} \mathbf{1 1}^{\prime}$ being the centering matrix. Then (9) is equivalent to

$$
\begin{aligned}
L_{\mathrm{MReg}}(c, \mathbf{w}) & =\|\mathbf{y}-c \mathbf{1}-\mathbf{J X} \mathbf{X}\|_{n^{-1}}^{2} \mathbf{1 1}^{\prime}+\|\mathbf{y}-c \mathbf{1}-\mathbf{J X} \mathbf{X}\|_{\mathbf{J}}^{2}+\lambda \mathbf{w}^{\prime} \mathbf{w} \\
& =\|\mathbf{y}-c \mathbf{1}\|_{n^{-1}}^{2} \mathbf{1 1}^{\prime}+\|\mathbf{J y}-\mathbf{J X} \mathbf{W}\|^{2}+\lambda \mathbf{w}^{\prime} \mathbf{w}
\end{aligned}
$$

where the norm notation is defined as $\|\mathbf{Z}\|_{\mathbf{A}}^{2}=\operatorname{tr} \mathbf{Z}^{\prime} \mathbf{A} \mathbf{Z}=\sum_{i=1}^{n} \sum_{j=1}^{n} \sum_{k=1}^{K} a_{i j}$ $z_{i k} z_{j k}$. Note that (10) is a decomposition in three terms with the intercept $c$ appearing alone in the first term so that it can be estimated independently of $\mathbf{w}$. The optimal $c$ in (10) equals $n^{-1} \mathbf{1}^{\prime} \mathbf{y}$. The remaining optimization of (10) in $\mathbf{w}$ simplifies into a standard ridge regression problem. Hence, the SVM with quadratic errors is equivalent to ridge regression. As the quadratic error has no hinge, even properly predicted objects with $q_{i}<-1$ for $i \in G_{-1}$ or $q_{i}>1$ for $i \in G_{1}$ can receive high error. In addition, the quadratic error is nonrobust, hence can be sensitive to outliers. Therefore, ridge regression is more restrictive than the quadratic hinge error and expected to give worse predictions in general.

\section{Optimal scaling and SVM}

Several ideas that are used in SVMs are not entirely new. In this section, we show that the application of optimal scaling known since the 1970s has almost the same aim as the SVM. Optimal scaling in a regression context goes back to the models MONANOVA (Kruskal 1965), ADDALS (Young et al. 1976a), MORALS (Young et al. 1976b), and, more recently, CatREG (Van der Kooij et al. 2006; Van der Kooij 2007). The main idea of optimal scaling regression (OS-Reg) is that a variable $\mathbf{y}$ is replaced by an optimally transformed variable $\widehat{\mathbf{y}}$. The regression loss function is not 


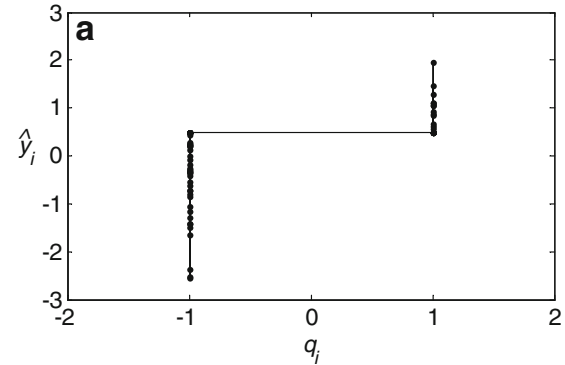

Optimal scaling transformation by primary approach to ties

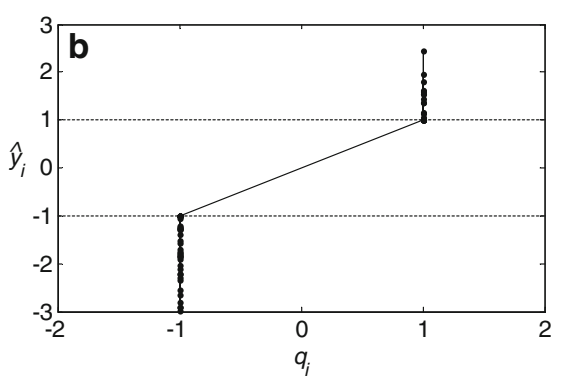

Optimal scaling transformation for SVMs

Fig. 3 Optimal scaling transformation $\widehat{\mathbf{y}}$ of the dependent variable $\mathbf{y}$. a An example transformation for the OS-Reg, b An example transformation for SVM

only optimized over the usual weights, but also over the optimally scaled variable $\widehat{\mathbf{y}}$. Many transformations are possible, see, for example, Gifi (1990). However, to make OS-Reg suitable for the binary classification problem, we use the so-called ordinal transformation with the primary approach to ties that allows to untie the tied data. This transformation was proposed in the context of multidimensional scaling to optimally scale the ordinal dissimilarities. As we are dealing with two groups only, this means that the only requirement is to constrain all $\widehat{y}_{i}$ in $G_{-1}$ to be smaller than or equal to all $\widehat{y}_{j}$ in $G_{1}$. An example of such a transformation is given in Fig. 3a.

OS-Reg can be formalized by minimizing

$$
L_{\mathrm{OS}-\operatorname{Reg}}(\widehat{\mathbf{y}}, \mathbf{w})=\sum_{i=1}^{n}\left(\widehat{y}_{i}-\mathbf{x}_{i}^{\prime} \mathbf{w}\right)^{2}+\lambda \mathbf{w}^{\prime} \mathbf{w}=\|\widehat{\mathbf{y}}-\mathbf{X} \mathbf{w}\|^{2}+\lambda \mathbf{w}^{\prime} \mathbf{w}
$$

subject to $\widehat{y}_{i} \leq \widehat{y}_{j}$ for all combinations of $i \in G_{-1}$ and $j \in G_{1}$ and $\widehat{\mathbf{y}}^{\prime} \widehat{\mathbf{y}}=n$. The latter requirement is necessary to avoid the degenerate zero-loss solution of $\widehat{\mathbf{y}}=\mathbf{0}$ and $\mathbf{w}=\mathbf{0}$. Without loss of generality, we assume that $\mathbf{X}$ is column centered here. In the usual formulation, no penalty term is present in (11), but here we add it because of ease of comparison with SVMs.

The error part of an SVM can also be expressed in terms of an optimally scaled variable $\widehat{\mathbf{y}}$. Then, the SVM loss becomes

$$
L_{\mathrm{SVM}-\mathrm{Abs}}(\widehat{\mathbf{y}}, \mathbf{w}, c)=\sum_{i=1}^{n}\left|\widehat{y}_{i}-\mathbf{x}_{i}^{\prime} \mathbf{w}-c\right|+\lambda \mathbf{w}^{\prime} \mathbf{w}
$$

subject to $\widehat{y}_{i} \leq-1$ if $i \in G_{-1}$ and $\widehat{y}_{i} \geq 1$ if $i \in G_{1}$. Clearly, for $i \in G_{-1}$ a zero error is obtained if $\mathbf{x}_{i}^{\prime} \mathbf{w}+c \leq-1$ by choosing $\widehat{y}_{i}=\mathbf{x}_{i}^{\prime} \mathbf{w}+c$. If $\mathbf{x}_{i}^{\prime} \mathbf{w}+c>-1$, then the restriction $\widehat{y}_{i} \leq-1$ becomes active so that $\widehat{y}_{i}$ must be chosen as -1 . Similar reasoning holds for $i \in G_{1}$, where $\widehat{y}_{i}=\mathbf{x}_{i}^{\prime} \mathbf{w}+c$ if $\mathbf{x}_{i}^{\prime} \mathbf{w}+c \geq 1$ (yielding zero error) and $\widehat{y}_{i}=1$ if $\mathbf{x}_{i}^{\prime} \mathbf{w}+c<1$. 
Just as the SVM, OS-Reg also has a limited number of support vectors. All objects $i$ that are below or above the horizontal line yield zero error. All objects $i$ that are have a value $\widehat{y}_{i}$ that is on the horizontal line generally give error, hence are support vectors.

The resemblances of SVM and OS-Reg is that both can be used for the binary classification problem, both solutions only use the support vectors, and both can be expressed in terms of an optimal scaled variable $\widehat{\mathbf{y}}$. Although, the SVM estimates the intercept $c$, OS-Reg implicitly estimates $c$ by leaving the position free where the horizontal line occurs, whereas the SVM attains this freedom by estimating $c$. One of the main differences is that OS-Reg uses squared error whereas SVM uses the absolute error. Also, in its standard form $\lambda=0$ so that OS-Reg does not have a penalty term. A final difference is that OS-Reg solves the degenerate zero loss solution of $\widehat{\mathbf{y}}=\mathbf{0}$ and $\mathbf{w}=\mathbf{0}$ by imposing the length constraint $\widehat{\mathbf{y}}^{\prime} \widehat{\mathbf{y}}=n$ whereas the SVM does this through setting a minimum difference of 2 between $\widehat{y}_{i}$ and $\widehat{y}_{j}$ if $i$ and $j$ are from different groups.

In some cases with $\lambda=0$, we found occasionally OS-Reg solutions where one of the groups collapsed at the horizontal line and the some objects of the other group were split into two points: one also at the horizontal line, the other at a distinctly different location. In this way, the length constraint is satisfied, but it is hardly possible to distinguish the groups. Fortunately, these solutions do not occur often and they never occurred with an active penalty term $(\lambda>0)$.

\section{SVM-Maj: a majorizing algorithm for SVM with robust hinge errors}

The SVM literature often solves the SVM problem by changing to the dual of (3) and expressing it as a quadratic program that subsequently is solved by special quadratic program solvers. A disadvantage of these solvers is that they may become computationally slow for large number of objects $n$ (although fast specialized solvers exist). Here, we use a different minimization approach based on iterative majorization (IM) algorithm applied to the primal SVM problem. One of the advantages of IM algorithms is that they guarantee descent, that is, in each iteration the SVM loss function is reduced until no improvement is possible. As the resulting SVM loss function for each of the three hinge errors is convex, the IM algorithm will stop when the estimates are sufficiently close to the global minimum. The combination of these properties forms the main strength of the majorization algorithm. In principle, a majorization algorithm can be derived for any error function that has a bounded second derivative as most robust errors have.

The general method of iterative majorization can be understood as follows. Let $f(\mathbf{q})$ be the function to be minimized. Then, iterative majorization makes use of an auxiliary function, called the majorizing function $g(\mathbf{q}, \overline{\mathbf{q}})$, that is dependent on $\mathbf{q}$ and the previous (known) estimate $\overline{\mathbf{q}}$. There are requirements on the majorizing function $g(\mathbf{q}, \overline{\mathbf{q}})$ : (1) it should touch $f$ at the supporting point $\mathbf{y}$, that is, $f(\overline{\mathbf{q}})=g(\overline{\mathbf{q}}, \overline{\mathbf{q}})$, (2) it should never be below $f$, that is, $f(\mathbf{q}) \leq g(\mathbf{q}, \overline{\mathbf{q}})$, and (3) $g(\mathbf{q}, \overline{\mathbf{q}})$ should be simple, preferably linear or quadratic in $\mathbf{q}$. Let $\mathbf{q}^{*}$ be such that $g\left(\mathbf{q}^{*}, \overline{\mathbf{q}}\right) \leq g(\overline{\mathbf{q}}, \overline{\mathbf{q}})$, for example, by choosing $\mathbf{q}^{*}=\arg \min _{\mathbf{q}} g\left(\mathbf{q}, \mathbf{q}^{*}\right)$. As the majorizing function is never 
below the original function, we obtain the so called sandwich inequality

$$
f\left(\mathbf{q}^{*}\right) \leq g\left(\mathbf{q}^{*}, \overline{\mathbf{q}}\right) \leq g(\overline{\mathbf{q}}, \overline{\mathbf{q}})=f(\overline{\mathbf{q}}) .
$$

This chain of inequalities shows that the update $\mathbf{q}^{*}$ obtained by minimizing the majorizing function never increases $f$ and usually decreases it. This constitutes a single iteration. By repeating these iterations, a monotonically nonincreasing (generally a decreasing) series of loss function values $f$ is obtained. For convex $f$ and after a sufficient number of iterations, the IM algorithm stops at a global minimum. More detailed information on iterative majorization can be found in De Leeuw (1994), Heiser (1995), Lange et al. (2000), Kiers (2002), and Hunter and Lange (2004) and an introduction in Borg and Groenen (2005).

An additional property of IM is useful for developing the algorithm. Suppose we have two functions, $f_{1}(\mathbf{q})$ and $f_{2}(\mathbf{q})$, and each of these functions can be majorized, that is, $f_{1}(\mathbf{q}) \leq g_{1}(\mathbf{q}, \overline{\mathbf{q}})$ and $f_{2}(\mathbf{q}) \leq g_{1}(\mathbf{q}, \overline{\mathbf{q}})$. Then, the function $f(\mathbf{q})=f_{1}(\mathbf{q})+f_{2}(\mathbf{q})$ can be majorized by $g(\mathbf{q})=g_{1}(\mathbf{q}, \overline{\mathbf{q}})+g_{2}(\mathbf{q}, \overline{\mathbf{q}})$ so that the following majorizing inequality holds:

$$
f(\mathbf{q})=f_{1}(\mathbf{q})+f_{2}(\mathbf{q}) \leq g_{1}(\mathbf{q}, \overline{\mathbf{q}})+g_{2}(\mathbf{q}, \overline{\mathbf{q}})=g(\mathbf{q}, \overline{\mathbf{q}})
$$

For notational convenience, we refer in the sequel to the majorizing function as $g(\mathbf{q})$ without the implicit argument $\overline{\mathbf{q}}$.

To find an algorithm, we need to find a majorizing function for (2). For the moment, we assume that a quadratic majorizing function exists for each individual error term of the form

$$
\begin{aligned}
f_{-1}\left(q_{i}\right) & \leq a_{-1 i} q_{i}^{2}-2 b_{-1 i} q_{i}+c_{-1 i}=g_{-1}\left(q_{i}\right) \\
f_{1}\left(q_{i}\right) & \leq a_{1 i} q_{i}^{2}-2 b_{1 i} q_{i}+c_{i}=g_{1}\left(q_{i}\right)
\end{aligned}
$$

Then, we combine the results for all terms and come up with the total majorizing function that is quadratic in $c$ and $\mathbf{w}$ so that an update can be readily derived. In the next subsection, we derive the SVM-Maj algorithm for general hinge errors assuming that (13) and (14) are known for the specific hinge error. In the appendix, we derive $g_{-1}\left(q_{i}\right)$ and $g_{1}\left(q_{i}\right)$ for the absolute, quadratic, and Huber hinge error SVM.

\subsection{The SVM-Maj algorithm}

Equation (2) was derived with the absolute hinge error in mind. Here, we generalize the definitions of the error functions $f_{-1}(q)$ and $f_{1}(q)$ in (2) to be any of the three hinge errors discussed above so that $L_{\mathrm{Q}-\mathrm{SVM}}$ and $L_{\mathrm{H}-\mathrm{SVM}}$ become special cases of $L_{\text {SVM }}$. For deriving the SVM-Maj algorithm, we assume that (13) and (14) are known for these hinge losses. Figure 4 shows that this is the case indeed. Then, let 

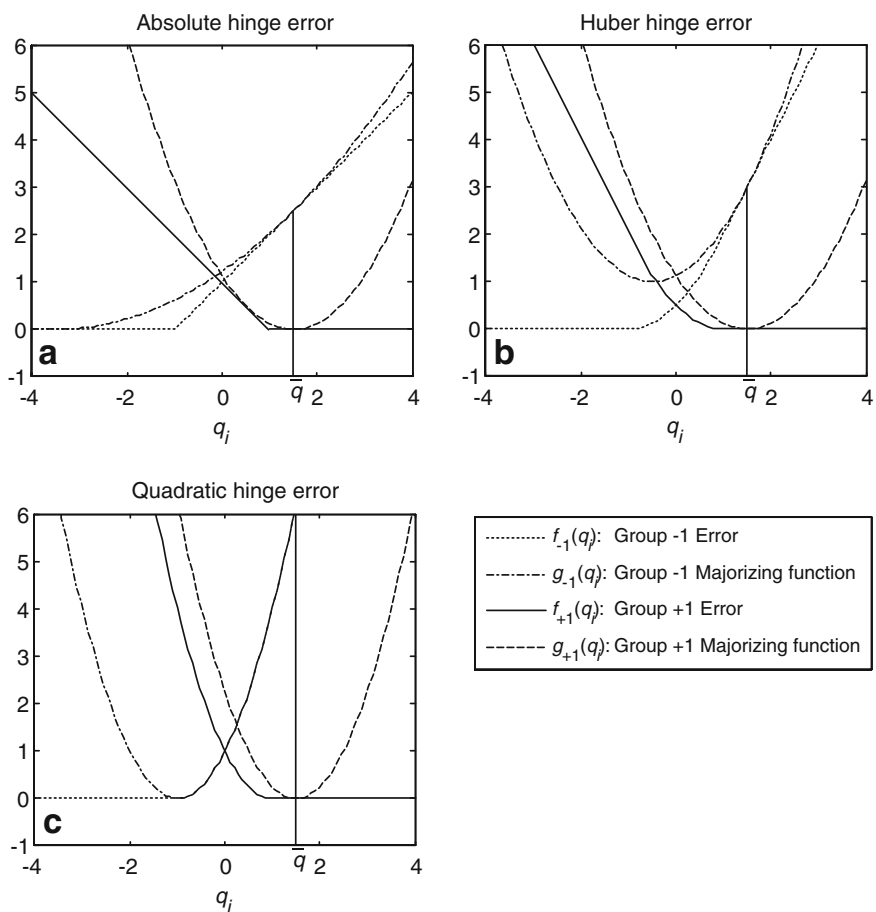

Fig. 4 Quadratic majorization functions for a the absolute hinge error, $\mathbf{b}$ the Huber hinge error, and $\mathbf{c}$ the quadratic hinge error. The supporting point is $\bar{q}=1.5$ both for the Group -1 and 1 error so that the majorizing functions touch at $q=\bar{q}=1.5$

$$
\begin{aligned}
& a_{i}= \begin{cases}\max \left(\delta, a_{-1 i}\right) & \text { if } i \in G_{-1}, \\
\max \left(\delta, a_{1 i}\right) & \text { if } i \in G_{1},\end{cases} \\
& b_{i}= \begin{cases}b_{-1 i} & \text { if } i \in G_{-1}, \\
b_{1 i} & \text { if } i \in G_{1},\end{cases} \\
& c_{i}= \begin{cases}c_{-1 i} & \text { if } i \in G_{-1}, \\
c_{1 i} & \text { if } i \in G_{1} .\end{cases}
\end{aligned}
$$

Summing all the individual terms leads to the majorization inequality

$$
L_{\mathrm{SVM}}(c, \mathbf{w}) \leq \sum_{i=1}^{n} a_{i} q_{i}^{2}-2 \sum_{i=1}^{n} b_{i} q_{i}+\sum_{i=1}^{n} c_{i}+\lambda \sum_{j=1}^{m} w_{j}^{2}
$$

It is useful to add an extra column of ones as the first column of $\mathbf{X}$ so that $\mathbf{X}$ becomes $n \times(m+1)$. Let $\mathbf{v}^{\prime}=\left[c \mathbf{w}^{\prime}\right]$ so that $q_{i}=c+\mathbf{x}_{i}^{\prime} \mathbf{w}_{i}$ can be expressed as $\mathbf{q}=\mathbf{X v}$. Then, (2) can be majorized as 


$$
\begin{aligned}
L_{\mathrm{SVM}}(\mathbf{v}) & \leq \sum_{i=1}^{n} a_{i}\left(\mathbf{x}_{i}^{\prime} \mathbf{v}\right)^{2}-2 \sum_{i=1}^{n} b_{i} \mathbf{x}_{i}^{\prime} \mathbf{v}+\sum_{i=1}^{n} c_{i}+\lambda \sum_{j=2}^{m+1} v_{j}^{2} \\
& =\mathbf{v}^{\prime} \mathbf{X}^{\prime} \mathbf{A} \mathbf{X} \mathbf{v}-2 \mathbf{v}^{\prime} \mathbf{X}^{\prime} \mathbf{b}+c_{m}+\lambda \mathbf{v}^{\prime} \mathbf{P} \mathbf{v} \\
& =\mathbf{v}^{\prime}\left(\mathbf{X}^{\prime} \mathbf{A} \mathbf{X}+\lambda \mathbf{P}\right) \mathbf{v}-2 \mathbf{v}^{\prime} \mathbf{X}^{\prime} \mathbf{b}+c_{m},
\end{aligned}
$$

where $\mathbf{A}$ is a diagonal matrix with elements $a_{i}$ on the diagonal, $\mathbf{b}$ is a vector with elements $b_{i}$, and $c_{m}=\sum_{i=1}^{n} c_{i}$, and $\mathbf{P}$ is the identity matrix except for element $p_{11}=0$. If $\mathbf{P}$ were $\mathbf{I}$, then the last line of (19) would be of the same form as a ridge regression. Differentating the last line of (19) with respect to $\mathbf{v}$ yields the system of equalities linear in $\mathbf{v}$

$$
\left(\mathbf{X}^{\prime} \mathbf{A} \mathbf{X}+\lambda \mathbf{P}\right) \mathbf{v}=\mathbf{X}^{\prime} \mathbf{b}
$$

The update $\mathbf{v}^{+}$solves this set of linear equalities, for example, by Gaussian elimination, or, less efficiently, by

$$
\mathbf{v}^{+}=\left(\mathbf{X}^{\prime} \mathbf{A} \mathbf{X}+\lambda \mathbf{P}\right)^{-1} \mathbf{X}^{\prime} \mathbf{b}
$$

Because of the substitution $\mathbf{v}^{\prime}=\left[c \mathbf{w}^{\prime}\right]$, the update of the intercept is $c^{+}=v_{1}$ and $w_{j}^{+}=v_{j+1}^{+}$for $j=1, \ldots, m$. The update $\mathbf{v}^{+}$forms the heart of the majorization algorithm for SVMs.

Extra computational efficiency can be obtained for the quadratic and Huber hinge errors for which $a_{-1 i}=a_{1 i}=a$ for all $i$ and this $a$ does not depend on $\overline{\mathbf{q}}$. In these cases, (21) simplifies into

$$
\mathbf{v}^{+}=\left(a \mathbf{X}^{\prime} \mathbf{X}+\lambda \mathbf{P}\right)^{-1} \mathbf{X}^{\prime} \mathbf{b}
$$

Thus, the $m \times n$ matrix $\mathbf{S}=\left(a \mathbf{X}^{\prime} \mathbf{X}+\lambda \mathbf{P}\right)^{-1} \mathbf{X}^{\prime}$ can be computed once and stored in memory, so that the update (21) simply amounts to setting $\mathbf{v}^{+}=\mathbf{S b}$. In this case, a single matrix multiplication of the $m \times n$ matrix $\mathbf{S}$ with the $n \times 1$ vector $\mathbf{b}$ is required to obtain an update in each iteration. Therefore, SVM-Maj for the Huber and quadratic hinge will be particularly efficient, even for large $n$ as long as $m$ is not too large.

The SVM-Maj algorithm for minimizing the SVM loss function in (2) is summarized in Algorithm 1. Note that SVM-Maj handles the absolute, quadratic, and Huber hinge errors. The advantages of SVM-Maj are the following. First, SVM-Maj approaches the global minimum closer in each iteration. In contrast, quadratic programming of the dual problem needs to solve the dual problem completely to have the global minimum of the original primal problem. Secondly, the progress can be monitored, for example, in terms of the changes in the number of misclassified objects. If no changes occur, then the iterations can be stopped. Thirdly, the computational time could be reduced, for example, by using smart initial estimates of $c$ and $\mathbf{w}$ available from a previous cross validation run. Note that in each majorization iteration a ridge regression problem is solved so that the SVM-Maj algorithm can be seen as a solution to the SVM problem via successive solutions of ridge regressions. 


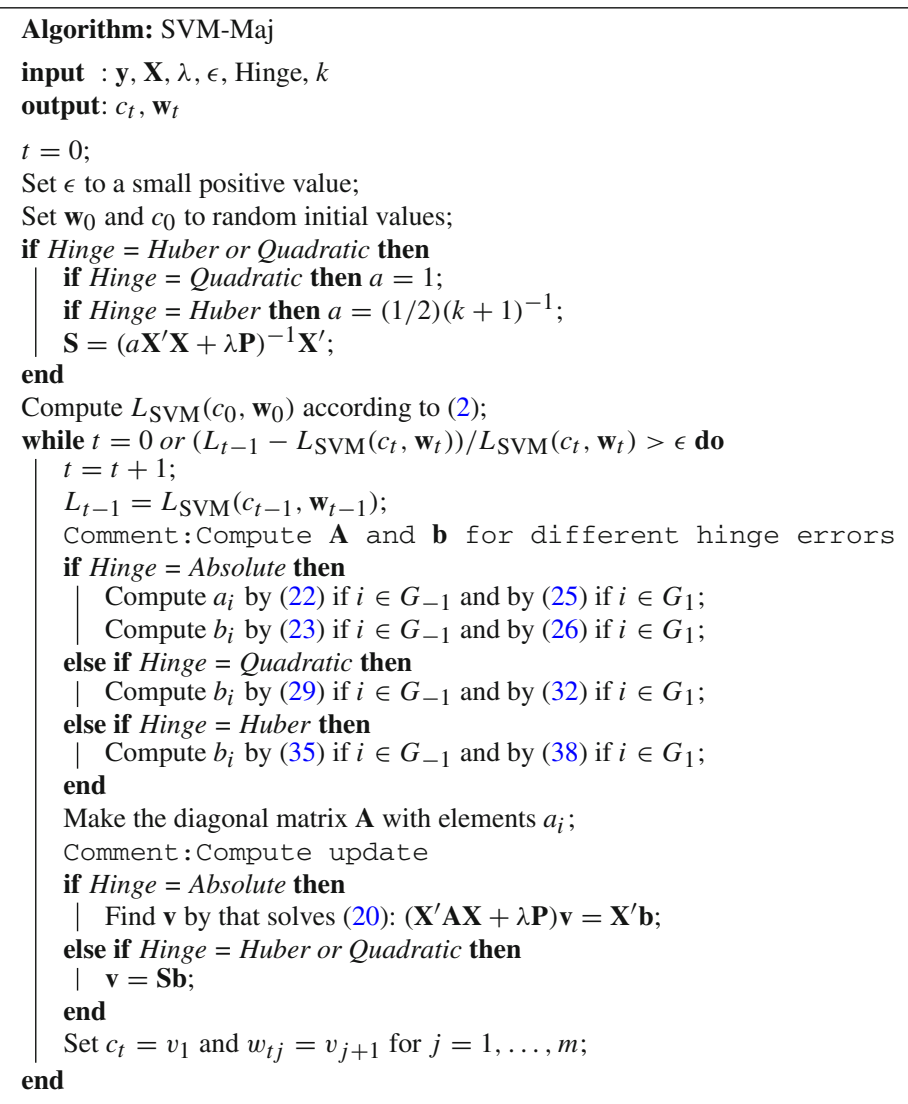

Algorithm 1: The SVM majorization algorithm SVM-Maj

A visual illustration of a single iteration of the SVM-Maj algorithm is given in Fig. 5 for the absolute hinge SVM. We fixed $c$ at its optimal value and the minimization is done only over $\mathbf{w}$, that is, over $w_{1}$ and $w_{2}$. Therefore, each point in the horizontal plane represents a combination of $w_{1}$ and $w_{2}$. In the same horizontal plane, the class 1 points are represented as open circles and the class -1 points as closed circles. The horizontal plane also shows the separation line and the margins corresponding to the current estimates of $w_{1}$ and $w_{2}$. It can be seen that the majorization function is indeed located above the original function and touches it at the dotted line, that is, at the current $w_{1}$ and $w_{2}$. At the location $\left(w_{1}, w_{2}\right)$ where this majorization function finds its minimum, $L_{\mathrm{SVM}}(c, \mathbf{w})$ is lower than at the previous estimate, so $L_{\mathrm{SVM}}(c, \mathbf{w})$ has decreased.

\section{Experiments}

To investigate the performance of the various variants of SVM algorithms, we report experiments on several data sets from the UCI repository (Newman et al. 1998) and 


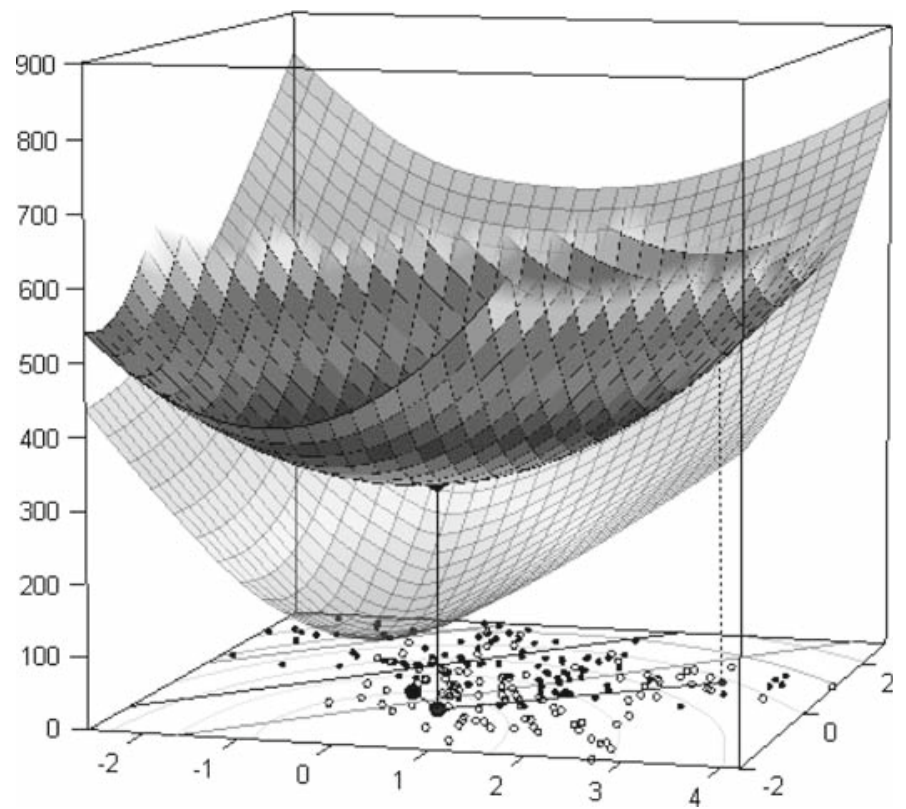

Fig. 5 Illustrative example of the iterative majorization algorithm for SVMs in action where $c$ is fixed and $w_{1}$ and $w_{2}$ are being optimized. The majorization function touches $L_{\mathrm{SVM}}(c, \mathbf{w})$ at the previous estimates of $\mathbf{w}$ (the dotted line) and a solid line is lowered at the minimum of the majorizing function showing a decrease in $L_{\mathrm{SVM}}(c, \mathbf{w})$ as well

the home page of LibSVM software (Chang and Lin 2006). These data sets cover a wide range of characteristics such as extent of being unbalanced (one group is larger than the other), number of observations $n$, ratio of observations to attributes $m / n$, and sparsity (the percentage of nonzero attribute values $x_{i j}$ ). More information on the data sets are given in Table 2 .

In the experiments, we applied the standard absolute hinge ( $\epsilon$-insensitive), the Huber hinge and quadratic hinge SVM loss functions. All experiments have been carried out in Matlab 7.2, on a $2.8 \mathrm{Ghz}$ Intel processor with $2 \mathrm{~GB}$ of memory under Windows XP. The performance of the majorization algorithms is compared to those of the off-theshelf programs LibSVM, BSVM (Hsu and Lin 2006), SVM-Light (Joachims 1999), and SVM-Perf (Joachims 2006). Although these programs can handle nonlinearity of the predictor variables by using special kernels, we limit our experiments to the linear kernel. Note that not all of these SVM-solvers are optimized for the linear kernel. In addition, no comparison between majorization is possible for the Huber hinge loss function as it is not supported by these solvers.

The numerical experiments address several issues. First, how well are the different hinge losses capable of predicting the two groups? Second, we focus on the performance of the majorization algorithm with respect to its competitors. We would like to know how the time needed for the algorithm to converge scales with the number of observations $n$, the strictness of the stopping criterion, and with $\lambda$; what is a suitable level for the stopping criterion. 
Table 2 Information on the 14 data sets used in the experiments

\begin{tabular}{llrrrrrr}
\hline Dataset & Source & \multicolumn{1}{l}{$n$} & $n_{1}$ & $n_{-1}$ & $m$ & Sparsity & Notes \\
\hline Australian & UCI & 690 & 307 & 383 & 14 & 20.04 & \\
Breast_cancer_w & UCI & 699 & 458 & 241 & 9 & 0.00 & \\
Heart_statlog & UCI & 270 & 120 & 150 & 13 & 0.00 & Standardized data \\
Hepatitis & UCI & 155 & 123 & 32 & 19 & 39.86 & \\
Sonar & UCI & 208 & 97 & 111 & 60 & 0.07 & \\
Voting & UCI & 434 & 167 & 267 & 16 & 45.32 & \\
Liver-disorders & LibSVM & 345 & 200 & 145 & 6 & 0.00 & \\
Liver-disorders2 & LibSVM & 345 & 200 & 145 & 6 & 0.92 & Scaled attributes \\
Diabetes & LibSVM & 768 & 500 & 268 & 8 & 0.00 & \\
Diabetes2 & LibSVM & 768 & 500 & 268 & 8 & 0.15 & Scaled attributes \\
Ionosphere & LibSVM & 351 & 225 & 126 & 34 & 11.59 & Scaled attributes \\
German.number & LibSVM & 1000 & 300 & 700 & 24 & 0.00 & \\
German.number2 & LibSVM & 1000 & 300 & 700 & 24 & 4.16 & Scaled attributes \\
Splice & LibSVM & 1000 & 517 & 483 & 60 & 0.00 & \\
\hline
\end{tabular}

$n_{1}$ and $n_{-1}$ are the number of observations with $y_{i}=1$ and $y_{i}=-1$, respectively. Sparsity equals the percentage of zeros in the data set. A data set with scaled attributes has maximum values +1 and minimum values -1

To answer these questions, we consider the following measures. First, we define convergence between two steps as the relative decrease in loss between two subsequent steps, that is, by $L_{\text {diff }}=\left(L_{t-1}-L_{t}\right) / L_{t}$. The error rate in the training data set is defined as the number of misclassified cases. To measure how well a solution predicts, we define the accuracy as the percentage correctly predicted out-of-sample cases in fivefold cross validation.

\subsection{Predictive performance for the three hinge errors}

It is interesting to compare the performance of the three hinge loss functions. Consider Table 3, which compares the fivefold cross-validation accuracy for the three different loss function. For each data set, we tried a grid of $\lambda$ values $\left(\lambda=2^{p}\right.$ for $p=-15,-14.5,-14, \ldots, 7.5,8$ where $2^{-15}=0.000030518$ and $2^{8}=256$ ). Alongside are given the values of the optimal $\lambda$ 's and times to convergence (stop whenever $L_{\text {diff }}<3 \times 10^{-7}$ ). From the accuracy, we see that there is no one best loss function that is suitable for all data sets. The absolute hinge is best in 5 of the cases, the Huber hinge is best in 4 of the cases, and the quadratic hinge is best in 7 of the cases. The total number is greater than 14 due to equal accuracies. In terms of computational speed, the order invariably is: absolute hinge is the slowest, Huber hinge is faster, and the quadratic hinge is the fastest.

The implementation of optimal scaling regression was also done in MatLab, but the update in each iteration for $\widehat{\mathbf{y}}$ by monotone regression using the primary approach to ties was calculated by a compiled Fortran subroutine. Therefore, the CPU time is 
Table 3 Performance of SVM models for the three hinge loss functions (Abs., Hub., and Quad.) and optimal scaling regression (OS)

\begin{tabular}{|c|c|c|c|c|c|c|c|c|c|c|c|c|}
\hline \multirow[t]{2}{*}{ Data set } & \multicolumn{4}{|c|}{ Optimal $p$} & \multicolumn{4}{|c|}{ CPU time in seconds } & \multicolumn{4}{|c|}{ Five-fold CV accuracy } \\
\hline & Abs. & Hub. & Quad. & OS & Abs. & Hub. & Quad. & OS & Abs. & Hub. & Quad. & OS \\
\hline Australian & -0.5 & 2.0 & 3.0 & -15.0 & 0.20 & 0.12 & 0.14 & 0.14 & 85.4 & 86.7 & 86.7 & 18.1 \\
\hline Breast_cancer_w & 7.5 & 6.0 & 8.0 & -15.0 & 0.13 & 0.11 & 0.04 & 0.49 & 96.7 & 96.6 & 96.7 & 97.7 \\
\hline Heart_statlog & 0.0 & 5.5 & 7.0 & 5.5 & 0.03 & 0.01 & 0.01 & 0.04 & 84.4 & 84.4 & 84.4 & 9.3 \\
\hline Hepatitis & 0.0 & 0.0 & 2.0 & -8.5 & 0.04 & 0.02 & 0.01 & 0.41 & 85.8 & 87.1 & 86.5 & 69.0 \\
\hline Sonar & 0.5 & 1.5 & 1.5 & -2.0 & 0.06 & 0.02 & 0.02 & 0.15 & 77.4 & 76.9 & 78.4 & 13.5 \\
\hline Voting & -5.5 & -1.5 & -0.5 & -0.5 & 0.46 & 0.11 & 0.10 & 0.35 & 97.0 & 96.8 & 97.0 & 6.2 \\
\hline Liver-disorders & 3.0 & 8.0 & 2.5 & 8.0 & 0.05 & 0.02 & 0.01 & 0.07 & 68.7 & 68.1 & 66.1 & 24.3 \\
\hline Liver-disorders 2 & -7.0 & -3.0 & 1.0 & -2.5 & 0.07 & 0.02 & 0.01 & 0.07 & 68.4 & 68.1 & 66.4 & 24.6 \\
\hline Diabetes & 1.0 & 0.5 & 3.5 & 7.5 & 0.24 & 0.04 & 0.01 & 0.07 & 77.3 & 78.0 & 77.6 & 79.0 \\
\hline Diabetes2 & -2.0 & -2.5 & 4.0 & 8.0 & 0.13 & 0.04 & 0.01 & 0.03 & 77.6 & 78.3 & 77.9 & 80.1 \\
\hline Ionosphere & -5.0 & 2.5 & -0.5 & -8.0 & 4.03 & 0.10 & 0.17 & 5.62 & 90.3 & 89.5 & 90.6 & 25.1 \\
\hline German.number & -0.5 & -4.0 & 3.5 & 3.5 & 1.71 & 0.17 & 0.03 & 0.18 & 77.2 & 77.0 & 77.3 & 26.2 \\
\hline German.number2 & 2.5 & 2.0 & -0.5 & 1.5 & 0.81 & 0.16 & 0.03 & 0.37 & 77.2 & 77.0 & 77.2 & 33.0 \\
\hline Splice & 5.0 & 7.0 & -0.5 & 3.5 & 3.21 & 0.34 & 0.14 & 0.61 & 80.7 & 81.4 & 81.1 & 13.0 \\
\hline
\end{tabular}

The optimal $\lambda=2^{p}$ is computed by fivefold cross validation. CPU-time to convergence for the optimal $\lambda$ and the prediction accuracy (in \%) is obtained for the 14 different test data sets from Table 2

not comparable to those of the other SVM methods that were solely programmed in MatLab. Optimal scaling regression performs well on three data sets (Breast cancer, Diabetes and Diabetes2) where the accuracy is better than the three SVM methods. On the remaining data sets, the accuracy is worse or much worse when compared to the SVM methods. It seems that in some cases OS regression can predict well, but its poor performance for the majority of the data sets makes it hard to use it as a standard method for the binary classification problem. One of the reasons for the poor performance could be due to solutions where all $\hat{y}_{i}$ s of one of the two classes collapses in the inequality constraint and the $\hat{y}_{i} s$ of the other class remain to have variance. In a transformation plot like Fig. 3 this situation means that the vertical scatter of either the -1 or 1 class collapses into a single point. By definition, the SVM transformation cannot suffer from this problem. More study is needed to understand if the collapse is the only reason for bad performance of OS-Reg, and, if possible, provide adaptations that make it work better for more data sets.

\subsection{Computational efficiency of SVM-Maj}

To see how computationally efficient the majorization algorithms are, two types of experiments were done. In the first experiment, the majorization algorithm is studied and tuned. In the second, the majorization algorithm SVM-Maj for the absolute hinge error is compared with several off-the-shelf programs that minimize the same loss function. 

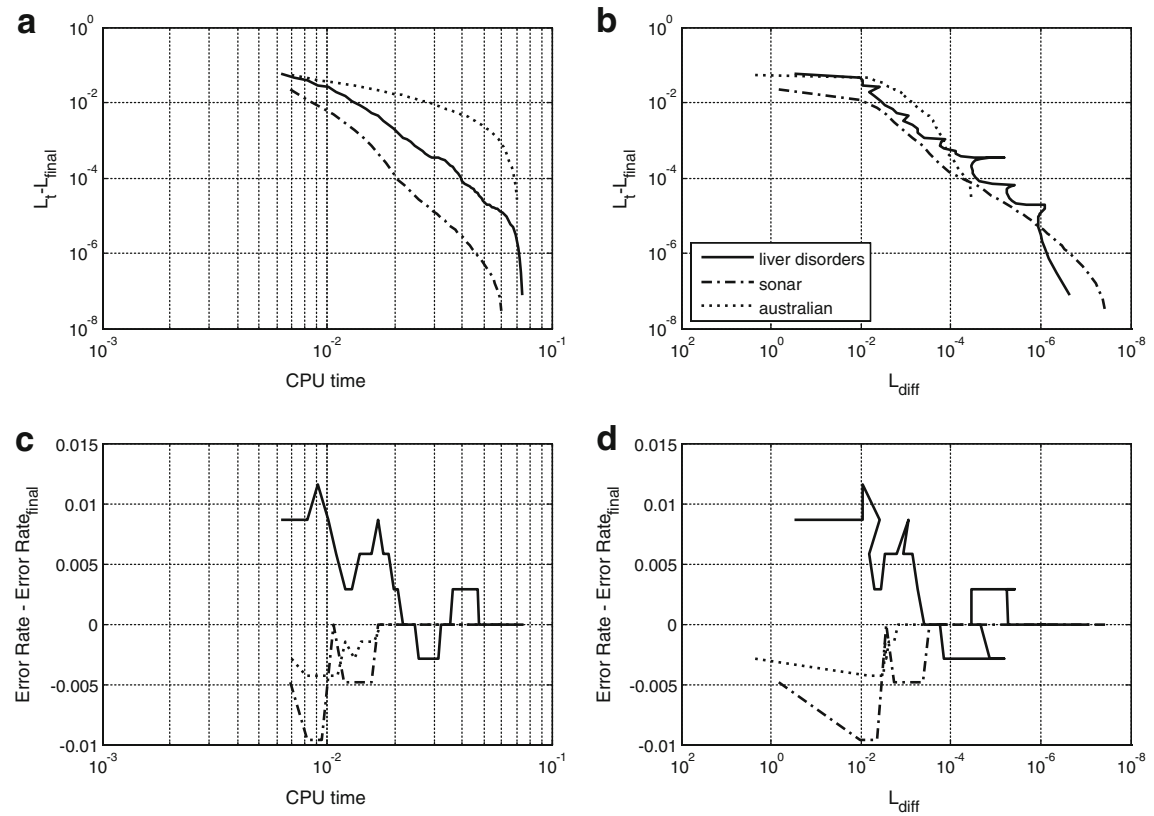

Fig. 6 The evolution of several statistics (see text for details) of three datasets: Australian (dotted lines), Sonar (dash-dot lines), and Liver Disorders (scaled, solid lines). Values of $\lambda$ 's are fixed at optimal levels for each dataset. Loss function used: absolute hinge

As the majorization algorithm is guaranteed to improve the $L_{\mathrm{SVM}}(c, \mathbf{w})$ in each iteration by taking a step closer to the final solution, the computational efficiency of SVM-Maj is determined by its stopping criterion. The iterations of SVM-Maj stop whenever $L_{\text {diff }}<\epsilon$. It is also known that majorization algorithms have a linear convergence rate (De Leeuw 1994), which can be slow especially for very small $\epsilon$. Therefore, we study the relations between four measures as they change during the iterations: (a) the difference between present and final loss, $L_{t}-L_{\text {final }}$, (b) the convergence $L_{\text {diff, }}$ (c) CPU time spent sofar, and (d) the difference between current and final within sample error rate.

Figure 6 shows the relationships between these measures for three exemplary data sets: Liver disorders, Sonar and Australian. Note that in Fig. 6c, d the direction of the horizontal axis is reversed so that in all four panels the right side of the horizontal axis means more computational investment. Figure 6 a draws the relationship between CPUtime and $L_{t}-L_{\text {final }}$, with $L_{\text {final }}$ the objective function values obtained at convergence with $\epsilon=3 \times 10^{-7}$. Notice that in most of the cases the first few iterations are responsible for the bulk of the decreases in the objective function values and most of the CPU time is spent to obtain small decreases in loss function values. Figure $6 \mathrm{~b}$ shows the relationship between $L_{t}-L_{\text {final }}$ and the convergence $L_{\text {diff }}$ that is used as a stopping criterion. The two lower panels show the development of the within sample error rate and CPU time (Fig. 6c) and convergence $L_{\text {diff }}$ (Fig. 6d). To evaluate whether it is worthwhile using a looser stopping criterion, it is in instructive to observe 


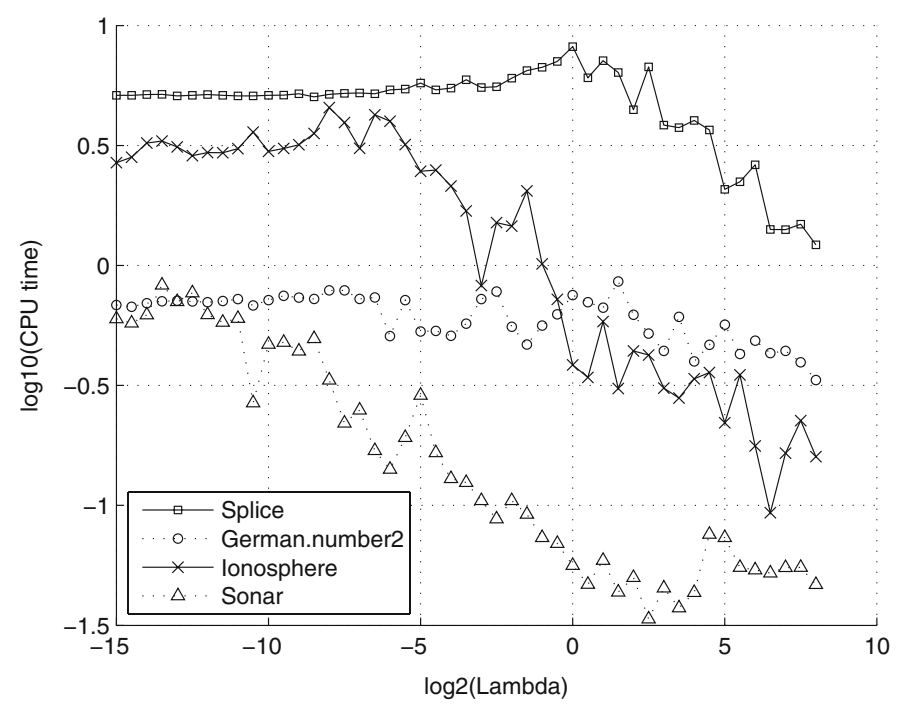

Fig. 7 The effect of changing $\lambda$ on CPU time taken by SVM-Maj to converge. Loss function used: absolute hinge

the path of the error rate over the iterations (the lower right panel). It seems that the error rate stabilizes for values of $L_{\text {diff }}$ below $10^{-6}$. Nevertheless, late-time changes sometimes occur in other data sets. Therefore, it does not seem recommendable to stop the algorithm much earlier, hence our recommendation of using $\epsilon=3 \times 10^{-} 7$.

The analogues of Figs. 6 and 7 were also produced for the Huber hinge and quadratic hinge loss functions. Overall, the same patterns as for the absolute hinge function can be distinguished, with several differences: the objective function decreases much faster (relative to CPU time), and the error rate stabilizes already at slightly greater values for the convergence criterion. In addition, the number of iterations until convergence by and large decline (vis-a-vis the absolute hinge function).

Figure 7 investigates how sensitive the speed of SVM-Maj is relative to changes in the values of $\lambda$ for four illustrative datasets (Splice, German-number with scaled attributed, Ionosphere, and Sonar). As expected, the relationship appears to be decreasing. Thus, for large $\lambda$ the penalty term dominates $L_{S V M}$ and SVM-Maj with the absolute hinge does not need too many iterations to converge. Note that the same phenomenon is in general observed for the other SVM-solvers as well so that, apparently, the case for large $\lambda$ is an easier problem to solve.

\subsection{Comparing efficiency of SVM-Maj with absolute hinge}

The efficiency of SVM-Maj can be compared with off-the-shelf programs for the absolute hinge error. As competitors of SVM-Maj, we use LibSVM, BSVM, SVMLight, and SVM-Perf. Note that the BSVM loss function differs from the standard SVM loss function by additionally penalizing the intercept. Nevertheless, we keep BSVM in our comparison to compare its speed against the others. We use the same 
14 data sets as before. As SVM-Maj, LibSVM, SVM-Light, and SVM-Perf minimize exactly the same loss function $L_{\mathrm{SVM}}$ they all should have the same global minimum. In addition to $L_{\mathrm{SVM}}$, the methods are compared on speed (CPU-time in seconds) at optimal levels of the $\lambda=2^{p}$ (or equivalent) parameter. Note that the optimal levels of $\lambda$ could differ slightly between methods as the off-the-shelf programs perform their own grid search for determining the optimal $\lambda$, that could be slightly different from those reported in Table 3. We note that the relationship between the $\lambda$ parameter in SVM-Maj and the $C$ parameter in LibSVM and SVM-light is given by $\lambda=0.5 / C$. For SVM-Maj, we choose three stopping criteria, that is, the algorithm is stopped whenever $L_{\text {diff }}$ is respectively smaller than $10^{-4}, 10^{-5}$, and $10^{-6}$.

For some data sets, it was not possible to run the off-the-shelf programs, sometimes because the memory requirements were too large, sometimes because no convergence was obtained. Such problems occurred for three data sets with SVM-Perf and two data sets with SVM-Light. Table 4 shows the results. Especially for $\epsilon=10^{-6}$, SVM-Maj gives solutions that are close to the best minimum found. Generally, Lib-SVM and SVM-Light obtain the lowest $L_{\text {SVM }}$. SVM-Maj performs well with $\epsilon=10^{-6}$, but even better values can be obtained by a stronger convergence criterion. Even though the loss function is slightly different, BSVM finds proper minima but is not able to handle all data sets. In terms of speed SVM-Maj is faster than its competitors in almost all cases. Of course, a smaller $\epsilon$ increases the CPU-time of SVM-Maj. Nevertheless, even for $\epsilon=0.0001$ good solutions can be found in a short CPU-time.

These results are also summarized in Fig. 8, where SVM-Maj is used with the default convergence criterion of $\epsilon=3 \times 10^{-7}$. As far as speed is concerned (see Fig. 8a), SVM-Maj ranks consistently amongst the fastest method. The quality of SVM-Maj is also consistently good as it has the same loss function as the global minimum with differences occurring less then 0.01 . Note that SVM-Perf finds consistently much higher loss function values than SVM-Maj, LibSVM and SVM-Light. Generally, the best quality solutions are obtained by LibSVM and SVM-Light although they tend to use more CPU time reaching it.

\section{Conclusions and discussion}

We have discussed how linear SVM can be viewed as a the minimization of a robust error function with a regularization penalty. The regularization is needed to avoid overfitting in the case when the number of predictor variables increases. We provided a new majorization algorithm for the minimization of the primal SVM problem. This algorithm handles the standard absolute hinge error, the quadratic hinge error, and the newly proposed Huber hinge error. The latter hinge is smooth everywhere yet is linear for large errors. The majorizing algorithm has the advantage that it operates on the primal, is easy to program, and can easily be adapted for robust hinge errors. We also showed that optimal scaling regression has several features in common with SVMs. Numerical experiments on fourteen empirical data sets showed that there is no clear difference between the three hinge errors in terms of cross validated accuracy. The speed of SVM-Maj for the absolute hinge error is similar or compares favorably to the off-the-shelf programs for solving linear SVMs. 


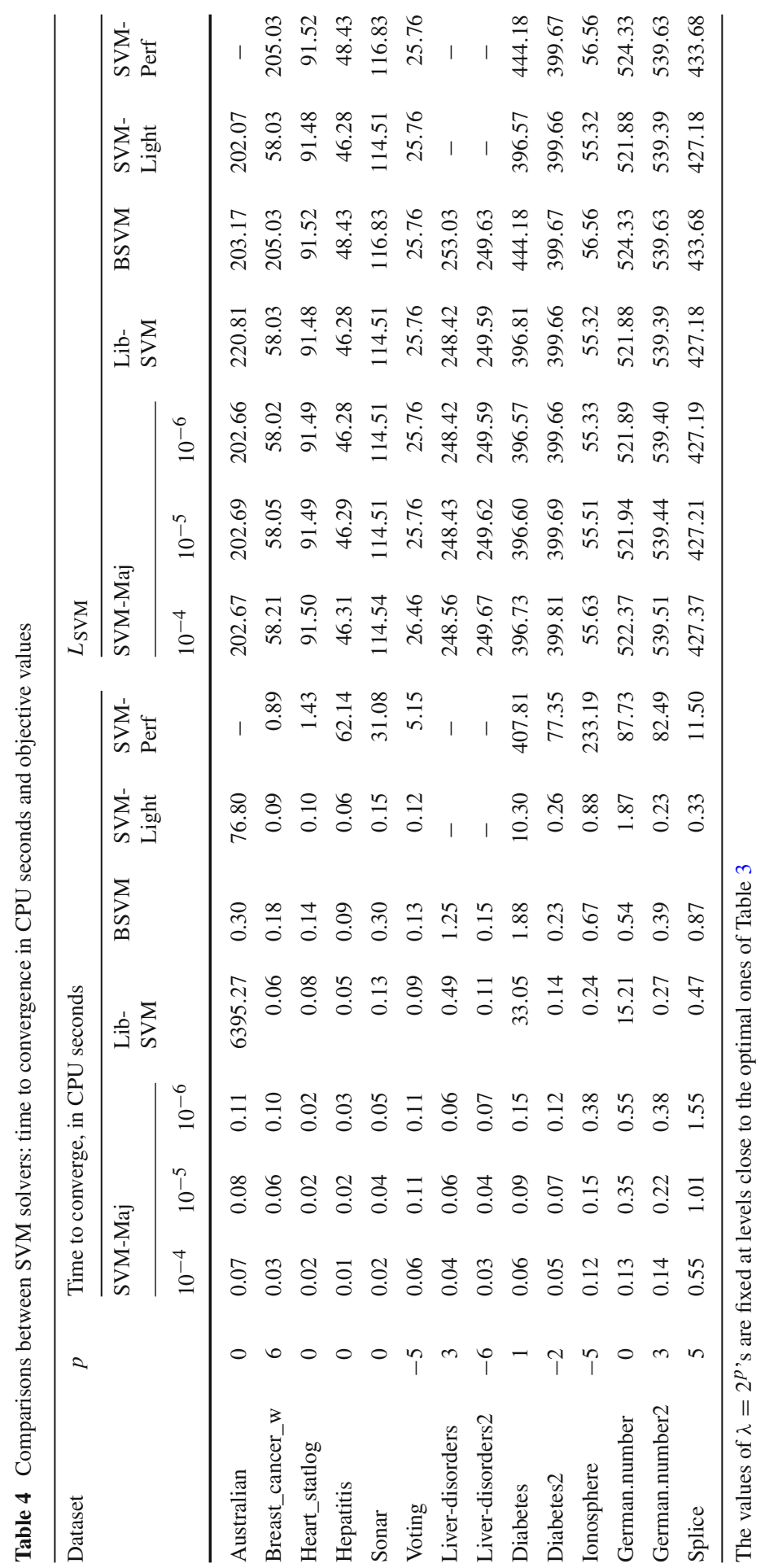


Fig. 8 Difference in performance of SVM algorithms with absolute hinge and SVM-Maj using $\epsilon=3 \times 10^{-7}$. a The CPU time used in seconds and $\mathbf{b}$ the difference of $L$ and the lowest $L$ amongst the methods
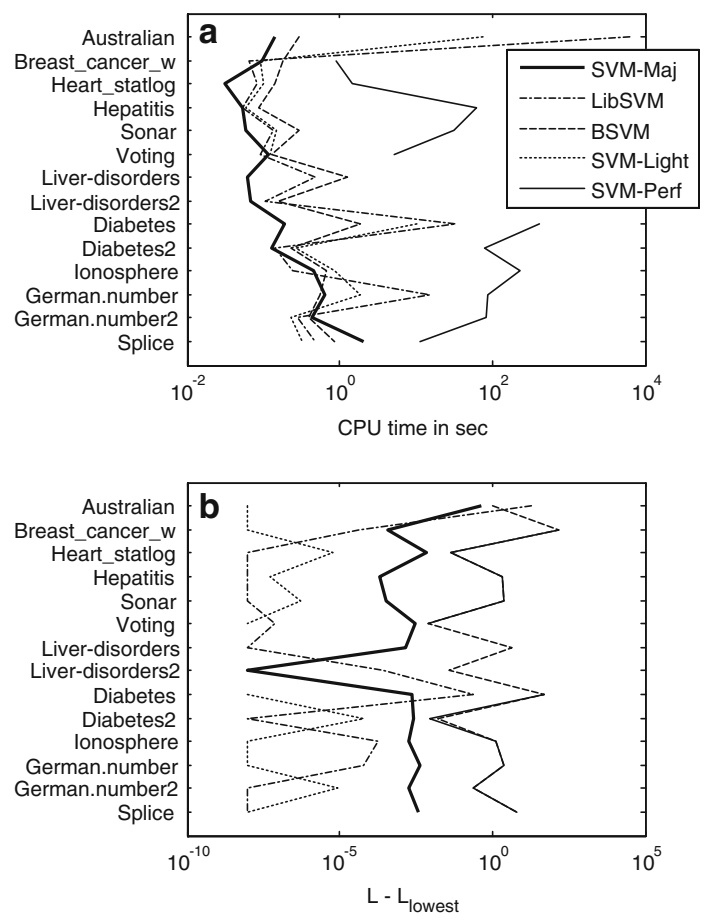

There are several open issues and possible extensions. First, the SVM-Maj algorithm is good for situations where the number of objects $n$ is (much) larger than the number of variables $m$. The reason is that each iteration solves an $(m+1) \times(m+1)$ linear system. As $m$ grows, each iteration becomes slower. Other majorization inequalities can be used to solve this problem yielding fast iterations at the cost of making (much) smaller steps in each iteration. A second limitation is the size of $n$. Eventually, when $n$ gets large, the iterations will become slow. The good thing about SVM-Maj is that each iteration is guaranteed to improve the SVM-Loss. The bad thing is that at most linear convergence can be reached so that for large $n$ one has to be satisfied with an approximate solution only. However, for $m$ not too large and even reasonably large $n$, SVM-Maj should work fine and be competitive. The SVM-Maj for the quadratic and Huber hinge are computationally more efficient than the absolute hinge, so they result in a faster algorithm, even for reasonably large $n$.

Second, this paper has focussed on linear SVMs. Nonlinearity can be brought in in two ways. In Groenen et al. (2007), we proposed to use optimal scaling for the transformation of the predictor variables. Instead of using kernels, we propose to use I-splines to accommodate nonlinearity in the predictor space. The advantage of this approach is that it can be readily applied in any linear SVM algorithm. The standard way of introducing nonlinearity in SVMs is by using kernels. We believe that this is also possible for SVM-Maj and intend to study this possibility in future publications. 
SVMs can be extended to problems with more than two classes in several ways. If the extension has error terms of the form $f_{1}(q)$ or $f_{-1}(q)$ then the present majorization results can be readily applied for an algorithm. We believe that applying majorization to SVMs is a fruitful idea that opens new applications and extensions to this area of research.

Open Access This article is distributed under the terms of the Creative Commons Attribution Noncommercial License which permits any noncommercial use, distribution, and reproduction in any medium, provided the original author(s) and source are credited.

\section{Appendix: Majorizing the hinge errors}

Here we derive the quadratic majorizing functions for the three hinge functions.

\section{A.1 Majorizing the absolute hinge error}

Consider the term $f_{-1}(q)=\max (0, q+1)$. For notational convenience, we drop the subscript $i$ for the moment. The solid line in Fig. 2a shows $f_{-1}(q)$. Because of its shape of a hinge, we have called this function the absolute hinge function. Let $\bar{q}$ be the known error $q$ of the previous iteration. Then, a majorizing function for $f_{-1}(q)$ is given by $g_{-1}(q, \bar{q})$ at the supporting point $\bar{q}=2$. We want $g_{-1}(q)$ to be quadratic so that it is of the form $g_{-1}(q)=a_{-1} q^{2}-2 b_{-1} q+c_{-1}$. To find $a_{-1}, b_{-1}$, and $c_{-1}$, we impose two supporting points, one at $\bar{q}$ and the other at $-2-\bar{q}$. These two supporting points are located symmetrically around -1 . Note that the hinge function is linear at both supporting points, albeit with different gradients. Because $g_{-1}(q)$ is quadratic, the additional requirement that $f_{-1}(q) \leq g_{-1}(q)$ is satisfied if $a_{-1}>0$ and the derivatives at the two supporting points of $f_{-1}(q)$ and $g_{-1}(q)$ are the same. More formally, the requirements are that

$$
\begin{aligned}
f_{-1}(\bar{q}) & =g_{-1}(\bar{q}), \\
f_{-1}^{\prime}(\bar{q}) & =g_{-1}^{\prime}(\bar{q}), \\
f_{-1}(-2-\bar{q}) & =g_{-1}(-2-\bar{q}), \\
f_{-1}^{\prime}(-2-\bar{q}) & =g_{-1}^{\prime}(-2-\bar{q}), \\
f_{-1}(q) & \leq g_{-1}(q) .
\end{aligned}
$$

It can be verified that the choice of

$$
\begin{aligned}
& a_{-1}=\frac{1}{4}|\bar{q}+1|^{-1}, \\
& b_{-1}=-a_{-1}-\frac{1}{4}, \\
& c_{-1}=a_{-1}+\frac{1}{2}+\frac{1}{4}|\bar{q}+1|,
\end{aligned}
$$


satisfies all these requirements. Figure 4 a shows the majorizing function $g_{-1}(q)$ with supporting points $\bar{q}=1.5$ as the dotted line.

For Class 1 , a similar majorizing function can be found for $f_{1}(q)=\max (0,1-q)$. However, in this case, we require equal function values and first derivative at $\bar{q}$ and at $2-\bar{q}$, that is, symmetric around 1 . The requirements are

$$
\begin{aligned}
f_{1}(\bar{q}) & =g_{1}(\bar{q}), \\
f_{1}^{\prime}(\bar{q}) & =g_{1}^{\prime}(\bar{q}), \\
f_{1}(2-\bar{q}) & =g_{1}(2-\bar{q}), \\
f_{1}^{\prime}(2-\bar{q}) & =g_{1}^{\prime}(2-\bar{q}), \\
f_{1}(q) & \leq g_{1}(q) .
\end{aligned}
$$

Choosing

$$
\begin{aligned}
& a_{1}=\frac{1}{4}|1-\bar{q}|^{-1} \\
& b_{1}=a_{1}+\frac{1}{4} \\
& c_{1}=a_{1}+\frac{1}{2}+\frac{1}{4}|1-\bar{q}|
\end{aligned}
$$

satisfies these requirements. The functions $f_{1}(q)$ and $g_{1}(q)$ with supporting points $\bar{q}=2$ or $\bar{q}=0$ are plotted in Fig. 4a.

Note that $a_{-1}$ is not defined if $\bar{q}=-1$. In that case, we choose $a_{-1}$ as a small positive constant $\delta$ that is smaller than the convergence criterion $\epsilon$ (introduced later). Strictly speaking, the majorization requirements are violated. However, by choosing $\delta$ small enough, the monotone convergence of the sequence of $L_{\mathrm{SVM}}(\mathbf{w})$ will be no problem. The same holds for $a_{1}$ if $\bar{q}=1$.

\section{A.2 Majorizing the quadratic hinge error}

The majorizing algorithm for the SVM with the quadratic hinge function is developed along the same lines as for the absolute hinge function. However, because of its structure, each iteration boils down to a matrix multiplication of a fixed $m \times n$ matrix with an $n \times 1$ vector that changes over the iterations. Therefore, the computation of the update is of order $O(\mathrm{~nm})$ which is more efficient than the majorizing algorithm for the absolute hinge error.

To majorize the term $f_{-1}(q)=\max (0, q+1)^{2}$ is relatively easy. For $\bar{q}>-1$, $f_{-1}(q)$ coincides with $(q+1)^{2}$. Therefore, if $\bar{q}>-1,(q+1)^{2}$ can be used to majorize $\max (0, q+1)^{2}$. Note that $(q+1)^{2} \geq 0$ so that $(q+1)^{2}$ also satisfies the majorizing requirements for $q<1$. For the case $\bar{q} \leq-1$, we want a majorizing function that has the same curvature as $(q+1)^{2}$ but touches at $\bar{q}$, which is obtained by the majorizing function $(q+1-(\bar{q}+1))^{2}=(q-\bar{q})^{2}$. Therefore, the majorizing 
function $g_{-1}=a_{-1} q^{2}-2 b_{-1} q+c_{-1}$ has coefficients

$$
\begin{aligned}
& a_{-1}=1, \\
& b_{-1}=\left\{\begin{array}{ll}
\bar{q} & \text { if } \bar{q} \leq-1 \\
-1 & \text { if } \bar{q}>-1
\end{array},\right. \\
& c_{-1}=\left\{\begin{array}{ll}
1-2(\bar{q}+1)+(\bar{q}+1)^{2} & \text { if } \bar{q} \leq-1 \\
1 & \text { if } \bar{q}>-1
\end{array} .\right.
\end{aligned}
$$

Similar reasoning can be held for $f_{1}(q)=\max (0,1-q)^{2}$ which has majorizing function $g_{1}=a_{1} q^{2}-2 b_{1} q+c_{1}$ and coefficients

$$
\begin{aligned}
& a_{1}=1, \\
& b_{1}=\left\{\begin{array}{ll}
1 & \text { if } \bar{q} \leq 1 \\
\bar{q} & \text { if } \bar{q}>1
\end{array},\right. \\
& c_{1}=\left\{\begin{array}{ll}
1 & \text { if } \bar{q} \leq 1 \\
1-2(1-\bar{q})+(1-\bar{q})^{2} & \text { if } \bar{q}>1
\end{array} .\right.
\end{aligned}
$$

Again, $a_{i}, b_{i}$, and $c_{i}$ are defined as in (15), (16), and (17), except that $\delta$ in (15) can be set to 0 , so that $a_{i}=1=a$ for all $i$.

\section{A.3 Majorizing the Huber hinge error}

The majorizing algorithm of the Huber hinge error function shares a similar efficiency as for the quadratic hinge: the coefficients $a_{1}$ and $a_{-1}$ are the same for all $i$, so that again an update boils down to a matrix multiplication of a matrix of order $m \times n$ with an $n \times 1$ vector.

To majorize $h_{-1}(q)$ we use the fact that the second derivative of $h_{-1}(q)$ is bounded. For $q \geq k, h_{-1}(q)$ is linear with first derivative $h_{-1}^{\prime}(q)=1$, so that its second derivative $h_{-1}^{\prime \prime}(q)=0$. For $q \leq-1, h_{-1}(q)=0$, so that here too $h_{-1}^{\prime \prime}(q)=0$. Therefore, $h_{-1}^{\prime \prime}(q)>0$ only exists for $-1<q<k$, where $h_{-1}^{\prime \prime}(q)=1$. Therefore, for $-1<q<k$, the quadratic majorizing function is equal to $h_{-1}(q)$, for $q \leq$ -1 and $q \geq k$, a quadratic majorizing function with the same second derivative of $(1 / 2)(k+1)^{-1}$ is produced that touches at the current estimate $\bar{q}$. Let the majorizing function $g_{-1}=a_{-1} q^{2}-2 b_{-1} q+c_{-1}$ has coefficients

$$
\begin{aligned}
& a_{-1}=(1 / 2)(k+1)^{-1}, \\
& b_{-1}= \begin{cases}a_{-1} \bar{q} & \text { if } \bar{q} \leq-1 \\
-a_{-1} & \text { if }-1<\bar{q}<k \\
a_{-1} \bar{q}-1 / 2 & \text { if } \bar{q} \geq k\end{cases} \\
& c_{-1}= \begin{cases}a_{-1} \bar{q}^{2} & \text { if } \bar{q} \leq-1 \\
a_{-1} & \text { if }-1<\bar{q}<k . \\
1-(k+1) / 2+a_{-1} \bar{q}^{2} & \text { if } \bar{q} \geq-k\end{cases}
\end{aligned}
$$


It may be verified for any $\bar{q}$ from the three intervals that $h_{-1}(\bar{q})=g_{-1}(\bar{q})$ and $h_{-1}^{\prime}(\bar{q})=g_{-1}^{\prime}(\bar{q})$ hold. In addition, $g_{-1}^{\prime \prime}(q)=(1 / 2)(k+1)^{-1} \geq h_{-1}^{\prime \prime}(q)$ for all $q$ (as long as $k>-1$ ) so that the second derivative $d_{-1}^{\prime \prime}(q)$ of the difference function $d_{-1}(q)=g_{-1}(q)-h_{-1}(q)$ equals $g_{-1}^{\prime \prime}(q)-h_{-1}^{\prime \prime}(q) \geq 0$ indicating that $d_{-1}(q)$ is convex. As $g_{-1}(q)$ touches $h_{-1}(q)$ at $\bar{q}, d_{-1}(\bar{q})=0$, so that, combined with convexity of $d_{-1}(q)$ the inequality $d_{-1}(q) \geq 0$ must hold implying the majorizing inequality $h_{-1}(q) \leq g_{-1}(q)$ for all $q$ with equality at $\bar{q}$.

For $h_{1}(q)$ similar reasoning can be held. Let the majorizing function $g_{1}=a_{-1} q^{2}-$ $2 b_{-1} q+c_{-1}$ has coefficients

$$
\begin{aligned}
& a_{1}=(1 / 2)(k+1)^{-1}, \\
& b_{1}= \begin{cases}1 / 2+a_{1} \bar{q} & \text { if } \bar{q} \leq-k \\
a_{1} & \text { if }-k<\bar{q}<1, \\
a_{1} \bar{q} & \text { if } \bar{q} \geq 1\end{cases} \\
& c_{1}= \begin{cases}1-(k+1) / 2+a_{-1} \bar{q}^{2} & \text { if } \bar{q} \leq-k \\
a_{1} & \text { if }-k<\bar{q}<1 . \\
a_{1} \bar{q}^{2} & \text { if } \bar{q} \geq 1\end{cases}
\end{aligned}
$$

Note that $a_{-1}$ and $a_{1}$ are exactly the same and both independent of $\bar{q}$. Therefore, the curvature of the majorizing functions for all Huber hinge errors is the same. This property is exploited in the simple update derived from (22).

\section{References}

Borg I, Groenen PJF (2005) Modern multidimensional scaling: theory and applications, 2nd edn. Springer, New York

Burges CJC (1998) A tutorial on support vector machines for pattern recognition. Knowl Discov Data Min 2:121-167

Chang C-C, Lin C-J (2006) LIBSVM: a library for support vector machines (Software available at http:// www.csie.ntu.edu.tw/ cjlin/libsvm)

Chu W, Keerthi S, Ong C (2003) Bayesian trigonometric support vector classifier. Neural Comput 15(9):2227-2254

Cristianini N, Shawe-Taylor J (2000) An introduction to support vector machines. Cambridge University Press, Cambridge

De Leeuw J (1994) Block relaxation algorithms in statistics. In: Bock H-H, Lenski W, Richter MM (eds) Information systems and data analysis. Springer, Berlin pp 308-324

Gifi A (1990) Nonlinear multivariate analysis. Wiley, Chichester

Groenen PJF, Nalbantov G, Bioch JC (2007) Nonlinear support vector machines through iterative majorization. In: Decker R, Lenz H-J (eds) Advances in data analysis. Springer, Berlin pp 149-162

Hastie T, Tibshirani R, Friedman J (2001) The elements of statistical learning. Springer, New York

Heiser WJ (1995) Convergent computation by iterative majorization: theory and applications in multidimensional data analysis. In: Krzanowski WJ (ed) Recent advances in descriptive multivariate analysis. Oxford University Press, Oxford pp 157-189

Hsu C-W, Lin C-J (2006) BSVM: bound-constrained support vector machines (Software available at http:// www.csie.ntu.edu.tw/ cjlin/bsvm/index.html)

Huber PJ (1981) Robust statistics. Wiley, New York

Hunter DR, Lange K (2004) A tutorial on MM algorithms. Am Stat 39:30-37

Joachims T (1999) Making large-scale SVM learning practical. In: Schölkopf B, Burges C, Smola A (eds) Advances in kernel methods—support vector learning. MIT-Press, Cambridge (http://www-ai. cs.uni-dortmund.de/DOKUMENTE/joachims_99a.pdf) 
Joachims T (2006) Training linear SVMs in linear time. In: Proceedings of the ACM conference on knowledge discovery and data mining (KDD) (http://www.cs.cornell.edu/People/tj/publications/ joachims_06a.pdf)

Kiers HAL (2002) Setting up alternating least squares and iterative majorization algorithms for solving various matrix optimization problems. Comput Stat Data Anal 41:157-170

Kruskal JB (1965) The analysis of factorial experiments by estimating monotone transformations of the data. J R Stat Soc Ser B 27:251-263

Lange K, Hunter DR, Yang I (2000) Optimization transfer using surrogate objective functions. J Comput Graph Stat 9:1-20

Newman D, Hettich S, Blake C, Merz C (1998) UCI repository of machine learning databases (http://www. ics.uci.edu/ mlearn/MLRepository.html University of California, Irvine, Department of Information and Computer Sciences)

Rosset S, Zhu J (2007) Piecewise linear regularized solution paths. Ann Stat 35:1012-1030

Rousseeuw PJ, Leroy AM (2003) Robust regression and outlier detection. Wiley, New York

Suykens JAK, Van Gestel T, De Brabanter J, De Moor B, Vandewalle J (2002) Least squares support vector machines. World Scientific, Singapore

Van der Kooij AJ (2007) Prediction accuracy and stability of regression with optimal scaling transformations. Unpublished doctoral dissertation, Leiden University

Van der Kooij AJ, Meulman JJ, Heiser WJ (2006) Local minima in categorical multiple regression. Comput Stat Data Anal 50:446-462

Vapnik VN (2000) The nature of statistical learning theory. Springer, New York

Young FW (1981) Quantitative analysis of qualitative data. Psychometrika 46:357-388

Young FW, De Leeuw J, Takane Y (1976) Additive structure in qualitative data: an alternating least squares method with optimal scaling features. Psychometrika 41:471-503

Young FW, De Leeuw J, Takane Y (1976) Regression with qualitative and quantitative variables: an alternating least squares method with optimal scaling features. Psychometrika 41:505-529 